\title{
calcul des pieux : tassements sous charge de service, frottement négatif
}

\author{
par \\ Marc Boulon \\ Maître Assistant à l'Université I de Grenoble \\ Jacques Desrues \\ Chercheur à l'Institut de Mécanique de Grenoble \\ et \\ Pierre Foray \\ Maître Assistant à l'École Nationale Supérieure d'Hydraulique de Grenoble
}

Exposé au Comité Français de Mécanique des Sols, séance du 14 mars 1977

\section{Introduction}

Le but poursuivi lors de cette étude est double :

- D'une part élaborer une méthode d'évaluation du tassement des pieux rendant compte au mieux des phénomènes observés lors d'essais de chargement statique au laboratoire ou in-situ. Ce calcul utilise la méthode des éléments finis.

- D'autre part, faire en sorte que ce calcul puisse servir d'outil pour le praticien désireux de déterminer rapidement un tassement de pieu.

Pour réaliser le premier point, on a besoin de modéliser aussi précisément que possible

- le comportement exact du sol sous les sollicitations exercées

- le mode de mise en place du pieu, et donc de la répartition initiale des contraintes

- les conditions de contact entre le sol et le pieu.

II faut donc introduire dans le calcul une loi de comportement correcte et des conditions aux limites réalistes. Plus on essaiera de décrire avec rigueur la réalité, et plus le calcul sera compliqué.

Mais si on veut aussi proposer une méthode de calcul pratique, on doit simplifier la réalité de façon à $n$ 'avoir à introduire qu'un nombre de paramètres limité, et facilement mesurables, par des essais de reconnaissance in-situ ou au laboratoire.

Dans ce souci, nous avons délibérément simplifié la loi de comportement du sol, en nous attachant par contre à bien décrire les conditions de contact sol-pieu. Ceci est justifié par les observations tirées de nombreux résultats d'essais de chargement statique, et des calculs publiés dans la littérature, qui montrent que le mécanisme de frottement latéral intéresse surtout la zone de contact entre le sol et le pieu et que la prise en compte du glissement relatif sol-pieu eșt aussi importante que l'hypothèse sur la loi de comportement du sol.

Au cours de cette étude, nous n'avons pas étudié le problème du tassement en fonction du temps, en ne nous attachant qu'au calcul des tassements finaux.

Dans une première partie, nous présentons l'étude du tassement des pieux sous charge de service, ainsi que des abaques de calcul pratique.
Dans une seconde partie, nous envisageons le problème du pieu sollicité en frottement négatif.

\section{Tassement sous charge verticale}

\subsection{Généralités}

L'élaboration d'une méthode de calcul réaliste doit être guidée en premier lieu par les nombreuses observations expérimentales faites sur le tassement des pieux. Les études menées par MM. Cambefort [8], Baguelin et al [2], Reese et al [15], Vesic et al [18], ainsi que les expériences que nous avons nous-mêmes réalisées en laboratoire [4], [5] et [11], montrent que les phénomènes prépondérants lors d'un essai de chargement de pieu sont :

- la non-linéarité et la non-réversibilité de la courbe charge-tassement (même dans le domaine des faibles déformations) dues à la fois au comportement du sol, à celui du pieu, et au mécanisme de mobilisation du frottement latéral.

- le comportement différent du pieu selon son mode de mise en place: courbe charge-tassement différente, mobilisation des contraintes de frottement latéral différente. Cette différence correspond à l'apparition de contraintes résiduelles existant dans le pieu avant chargement, dues à la mise en place ou à un cycle de chargement précédent.

Par ailleurs, des mesures de déformation du sol faites au voisinage du pieu montrent que, lors d'un essai de tassement, la mobilisation du frottement latéral intéresse un très faible volume de sol autour du fat. II s'agit donc d'un phénomène essentiellement lié aux conditions d'interface sol-pieu et qui justifie l'utilisation en première approximation d'une loi de comportement élastique pour le sol.

De nombreuses méthodes élastiques ont ainsi vu le jour, qui diffèrent par le principe du calcul.

Celles des "fonctions de transfert 》 (Reese [15]) assimilant l'action du sol sur le pieu en un point à celle de ressorts, constituent une élasticité simplifiée, à rapprocher de la méthode semi-empirique de Cambefort [8] développée par Cassan [9].

Les premiers calculs rigoureux en élasticité ont été ceux utilisant les formules de Mindlin qui donnent les 
contraintes et les déplacements en tout point d'un massif élastique semi-infini, crés par une force ponctuelle agissant à l'intérieur du massif et qui sont l'équivalent des formules de Boussinesq pour les fondations superficielles. L'intégration numérique de ces formules avec les conditions aux limites du pieu a été faite par Thurman et d'Appolonia en 1965 [17], puis développée par Poulos et al [14] et Butterfield et Bannerjee [7].

L'énorme avantage de ces calculs a été de pouvoir établir des abaques donnant le tassement du pieu en fonction de sa géométrie, de sa compressibilité relative par rapport à celle du sol et des caractéristiques élastiques du sol. Le cas des bicouches a été également envisagé par ces auteurs.

Les calculs de tassements les plus récents utilisent la méthode des éléments finis. Celle-ci permet d'introduire des conditions aux limites complexes pour le problème et se prête donc particulièrement bien aux cas réels. De plus, on peut y utiliser des lois de comportement du sol plus réalistes que l'élasticité.

Citons l'introduction du glissement à l'interface dans les travaux de Thurman et d'Appolonia [17], Ellison et al [12] et Zinebi [20].

Frank [13] propose la superposition de la dilatance à l'élasticité classique dans le cas du pieu "collé ". Celle-ci n'a que des effets minimes sur le comportement du pieu en petites déformations.

Actuellement la méthode des éléments finis permet de simuler le chargement d'un pieu dans un domaine de déformations plus importants en prenant des lois du type élasto-plastique (Ellison et al [12]), hyperbolique (Desai [10]), visco-élastique (Booker-Poulos [3]) ou incrémentale, comme cela a été réalisé à Grenoble (Boulon et al [5]).

\subsection{Calcul réalisé}

\subsubsection{Méthode}

Dans le calcul présenté ici, qui utilise la méthode des éléments finis, on s'est attaché :

- D'une part à simuler correctement le glissement progressif du sol au contact du fût du pieu, en introduisant un frottement de type Coulomb à l'interface. L'expérience montre en effet que l'intervention du glissement est prépondérante dans la modification de la répartition des contraintes de frottement latéral le long du pieu.

- D'autre part, à faire en sorte que ce calcul puisse être effectué pour un grand nombre de cas, et sans trop de frais, de façon à établir des abaques pour les cas rencontrés le plus couramment. On a donc préféré ici s'en tenir à la loi de comportement la plus simple pour le sol, l'élasticité linéaire isotrope. On initialise le calcul en admettant ici que la répartition des contraintes normales le long du pieu est triangulaire, égale à la poussée des terres au repos,

$$
\sigma_{n}=k_{\circ} \gamma z
$$

et qu'il n'y a pas de contraintes tangentielles.

Le principe du calcul consiste à appliquer au pieu un incrément de déplacement et à recalculer la charge en tête qui l'a produit. Au départ, on considère que tous les points du sol suivent les déplacements du pieu, c'est-à-dire qu'on suppose le sol "collé au pieu". On détermine ainsi la dissipation de l'effort dans le pieu et la valeur des contraintes de frottement latéral aux points nodaux situés à l'interface sol-pieu.

Au fur et à mesure que la charge en tête augmente, ces contraintes augmentent, et à partir d'un certain moment elles vont atteindre en certains points nodaux la valeur limite donnée par la loi de frottement:

$\tau \leqslant \tau_{\max }=\sigma_{\mathrm{n}} \operatorname{tg} \delta$ dans le cas d'un sol pulvérulent, $\delta$ étant l'angle de frottement pieu-sol

$\tau \leqslant \tau_{\max }=\mathrm{C}_{\mathrm{a}}$ dans le cas d'un sol argileux, $\mathrm{C}_{\mathrm{a}}$ étant l'adhérence sol-pieu $\mathrm{C}_{\mathrm{a}}=\alpha \mathrm{C}_{u}$

En ces points, il y a donc glissement du sol par rapport au pieu. La méthode permet de le calculer. Ces glissements modifient considérablement la répartition des contraintes tangentielles le long du fût. Lorsqu'on continue à augmenter la charge en tête, tous les points nodaux de l'interface finissent par glisser.
L'algorithme général du frottement-glissement est présenté dans un article plus spécialisé [6].

\subsubsection{Résultats}

Nous présentons les résultats obtenus pour le tassement dans les 2 cas suivants:

- Pieu sollicité en chargement croissant : c'est le cas qui peut simuler le problème d'un pieu moulé, c'est-à-dire n'ayant pas été sollicité lors de sa mise en place.

- Pieu subissant des sollicitations alternéès, c'est-à-dire un chargement suivi d'un déchargement, puis d'un rechargement. $\mathrm{Ce}$ dernier cas est particulièrement intéressant puisqu'il permet une approche des phénomènes d'apparition des contraintes résiduelles dans le pieu lors de sa mise en place par fonçage ou battage.

\section{Pieu soumis à un chargement croissant}

\section{Courbe charge-tassement}

La figure 1 représente un exemple de courbe chargetassement calculée pour un pieu d'élancement $D / B=15$ fiché dans un sable moyennement dense, de module d'Youn $E=\mathbf{5 0 0}$ bars, et dont la compressibilité relative par rapport au sol est de $1000\left(K=E_{\text {pied }} / E_{\text {so1 }}=1000\right)$. Les caractéristiques d'interface sont celles qui ont été mesurées au laboratoire (frottement sable-béton): $\operatorname{tg} \delta=0,5$

\section{Influence du glissement}

On remarque en premier lieu l'incurvation de la courbe : le tassement $n$ 'augmente pas linéairement avec la charge. La courbe devient néanmoins sensiblement linéaire après un certain point marqué en noir. $\mathrm{Ce}$ point correspond au moment où tous les points nodaux de l'interface sol-fût du pieu ont glissé. On distingue donc trois phases dans la courbe charge-tassement :

- Le premier pas correspond au moment où tous les points du pieu sont « collés ». C'est une partie parfaitement linéaire, on l'a prolongée sur la figure.

- La partie incurvée correspond au glissement progressif des points du fât.

- La dernière partie, sensiblement linéaire, correspond au tassement après glissement complet le long du fût.

Le calcul met ici en évidence le fait important que la non-línéarité de la courbe charge-tassements dans le domaine des petites déformations provient en premier lieu du glissement du sol le long du fût, et non pas d'emblée de la non-linéarité du comportement du sol.

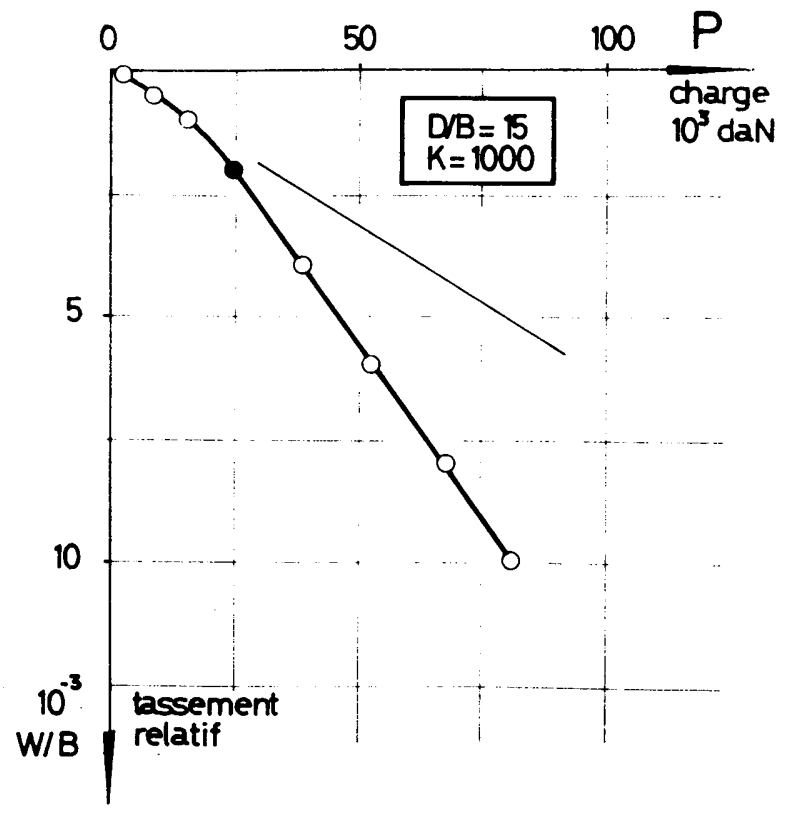

Fig. 1 Courbe de charge-tassement calculée : influence du glissement

$D:$ longueur du pieu $B$ : diamètre $w$ : tassement 


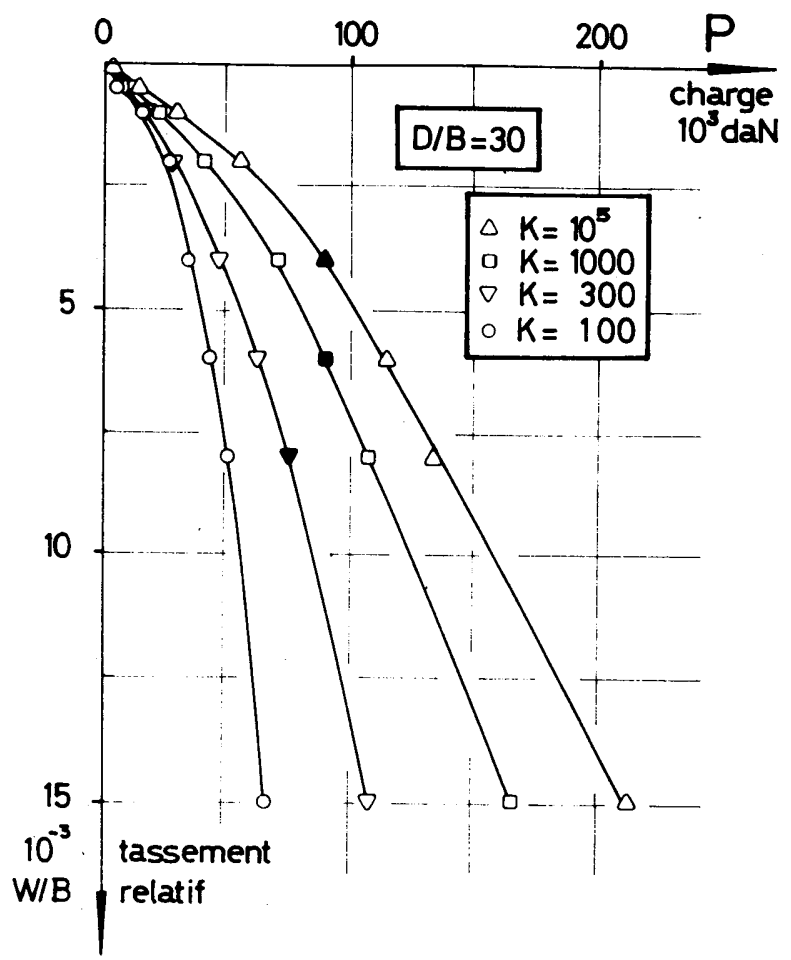

Fig. 2 Evolution de la courbe charge-tassement calculée en fonction de la compressibilité relative du pieu par rapport au sol

Influence de la compressibilité du pieu

Celle-ci peut être caractérisée par le rapport $K$ du module du pieu à celui du sol. La figure 2 présente les courbes charge-tassements calculées pour des pieux identiques géométriquement, mais de modules différents.

On vérifie que plus le pieu est compressible par rapport au sol, plus le tassement est fort.

On observe de plus que, plus le pieu est compressible, plus le glissement complet est retardé, la partie incurvée est plus grande.

Influence de la longueur du pieu et effet d'échelle

Pour un même diamètre, la longueur du pieu a un effet

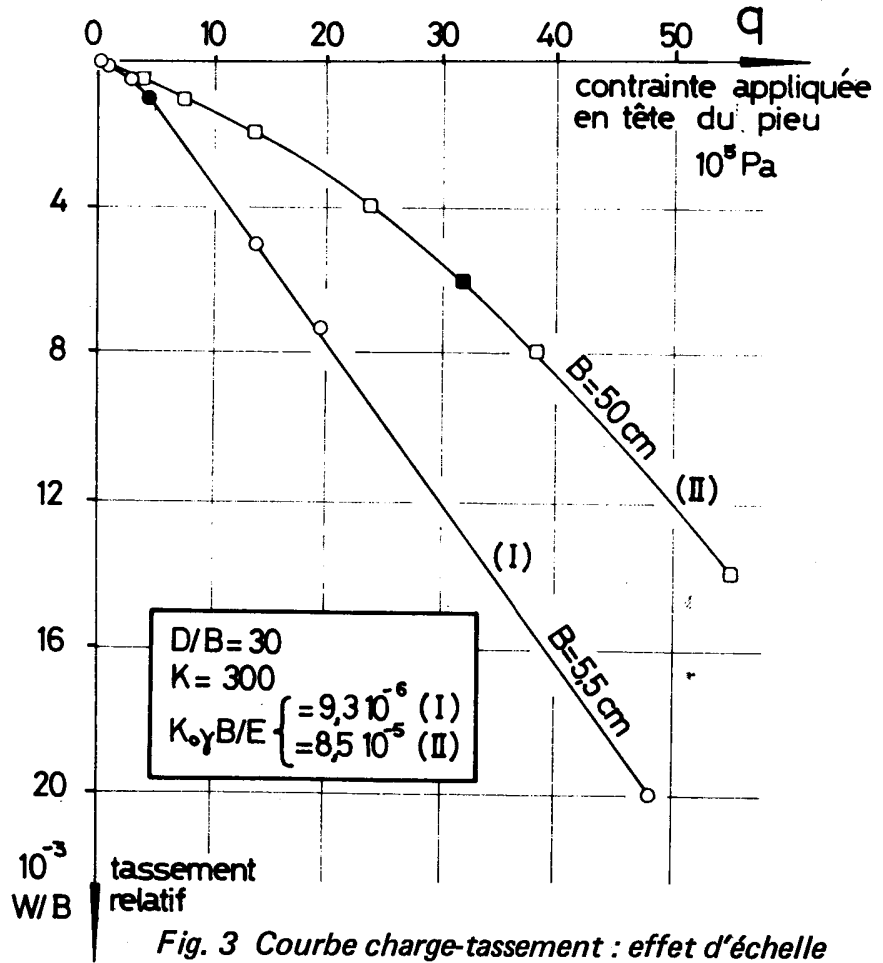

important sur le tassement, essentiellement par une extension de la partie incurvée de la courbe chargetassements.

Une autre constatation importante dont rend compte le calcul avec glissement est que deux pieux homothétiques n'ont pas le même comportement. En effet, pour le pieu le plus grand, les contraintes normales absolues le long du fât seront plus fortes, et par conséquent le glissement se produira plus tard. La figure 3 illustre cette différence, la variation d'échelle étant caractérisée par le paramètre

$$
\frac{K \circ \gamma B}{E}
$$

avec $\mathrm{K}_{\mathrm{o}}$ coefficient de poussée des terres au repos

$\gamma$ poids volumique du terrain latéral

B diamètre du pieu

E module d'Young du sol

Cette figure montre combien il serait illusoire de transposer quantitativement les résultats d'essais de tassements effectués au laboratoire à un cas réel in-situ.

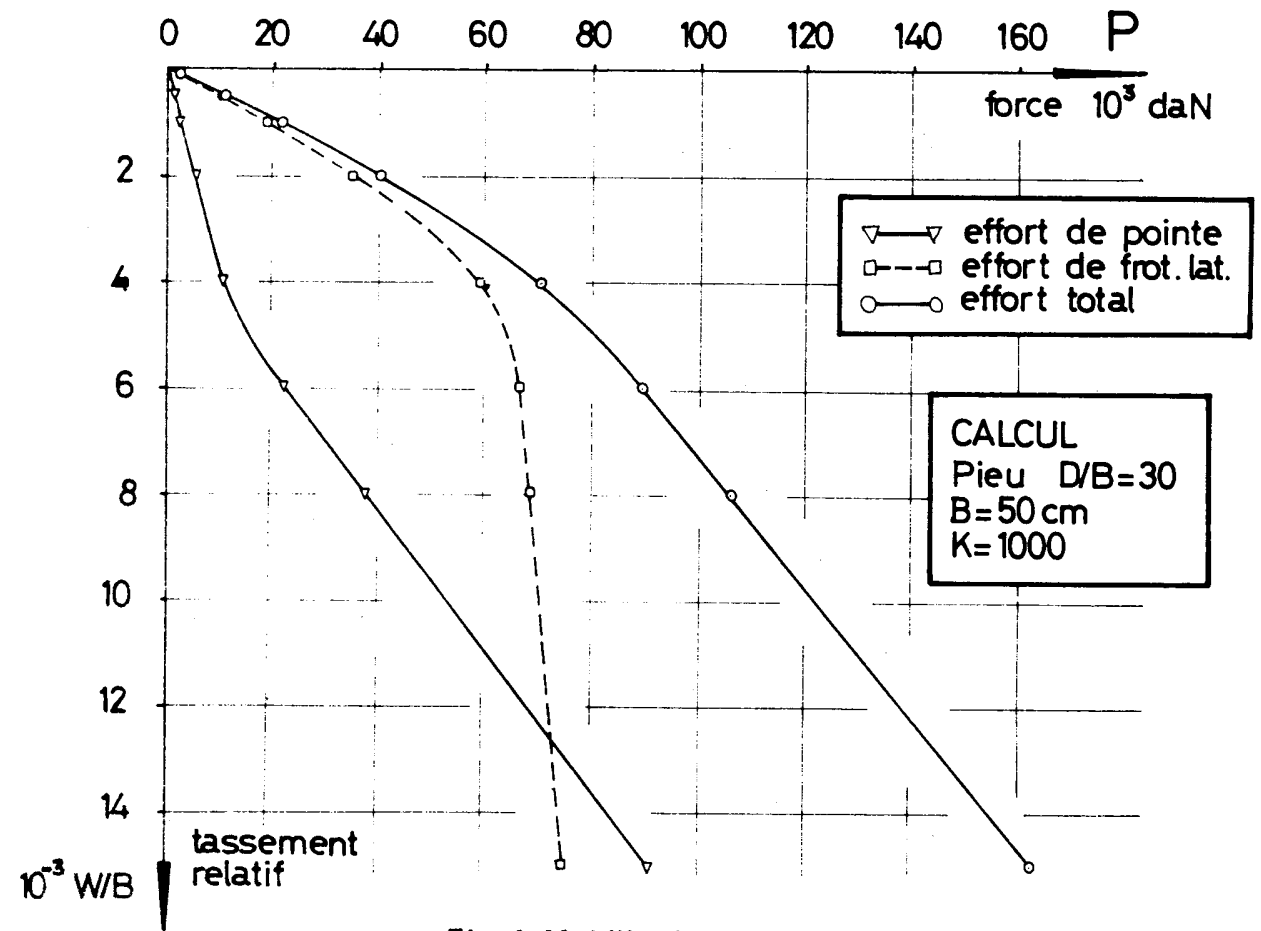

Fig. 4 Mobilisation des efforts 
Mobilisation des efforts

La figure 4 représente les courbes calculées de mobilisation des efforts pour un pieu réel. On a porté l'effort total appliqué en tête, l'effort de pointe et l'évolution du frottement latéral.

On vérifie très bien les phénomènes suivants observés expérimentalement :

- le frottement latéral se mobilise très rapidement pour atteindre un maximum au-delà duquel il augmente très peu,

- la courbe de mobilisation de l'effort de pointe présente une courbure opposée à celle de la courbe de frottement latéral : la pointe se met à augmenter fortement lorsque le frottement latéral atteint son maximum.

Le calcul montre que le maximum du frottement latéral correspond au glissement total du sol le long du fût, à partir de ce moment, la charge se reporte sur la pointe. II faut noter qu'un calcul "collé " ne peut pas rendre compte de façon correcte de ces débuts de courbes.

La forme des courbes de mobilisation des efforts est affectée par la compressibilité du pieu et son élancement.

Répartition du frottement latéral

La figure 5 présente les répartitions de la contrainte de cisaillement calculée le long du fât. Le pieu calculé est un pieu grandeur réelle, d'élancement $D / B=20$ et de compressibilité $K=300$. Le terrain est un sable dense. La formulation adimensionnelle en $\sigma \pi \mathrm{BD} / \mathrm{P}$ ( $\mathrm{P}$ charge en tête) permet de bien suivre l'évolution du phénomène.

II apparaît nettement que le frottement latéral, initialement concentré en haut du pieu, voit sa répartition se modifier et évoluer progressivement vers une prépondérance de la partie basse.

Les courbes de répartition du frottement latéral sont, toutes choses égales par ailleurs, influencées par la compressibilité relative du pieu et son élancement : le pieu compressible « ignore » les déplacements faibles appliqués en tête, du fait du raccourcissement élastique du fat qui fait se reporter la charge principalement aux points du haut du pieu. Le pieu rigide, au contraire, se déplace à peu près de la même quantité sur toute sa hauteur ; la mobilisation du frottement à tous les niveaux est donc simultanée sur tout le fût.

Ce fait est illustré par les figures 6 et 7 pour lesquelles on a tracé, pour différents niveaux le long du fât, la contrainte de cisaillement (moyenne) locale en fonction du

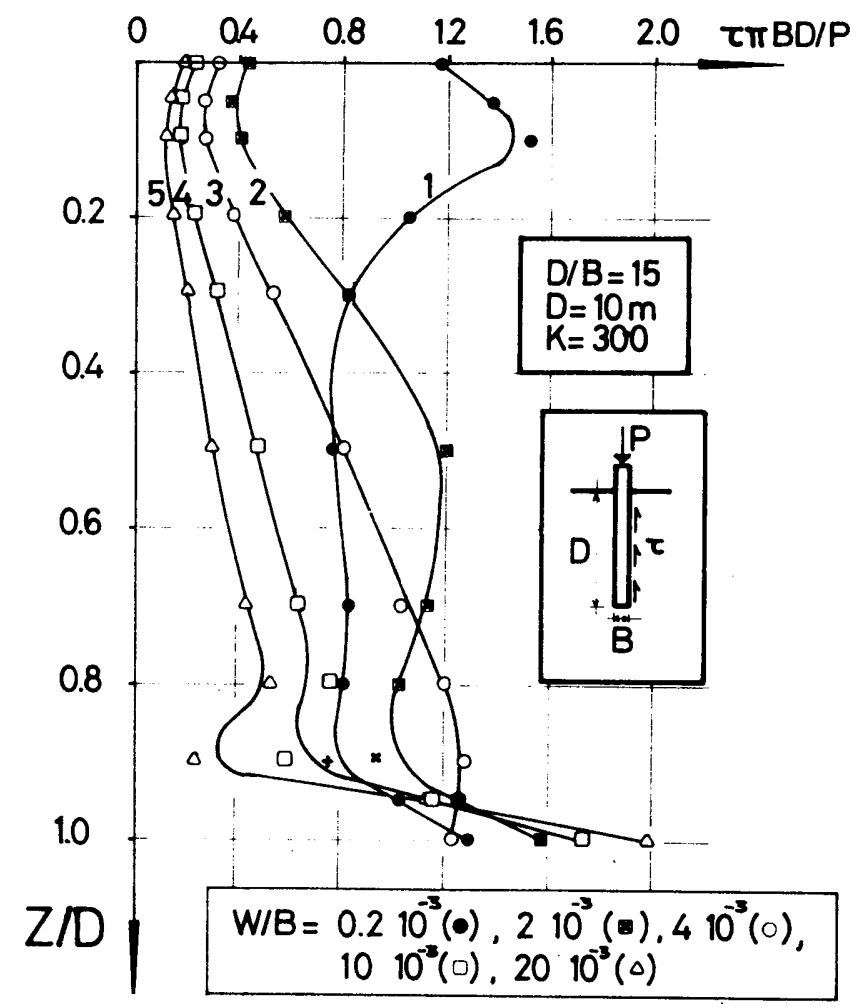

Fig. 5 Répartition calculée de la contrainte de cisaillement le long du fût

déplacement de la tête du pieu (et non pas au point considéré).

Comparaison avec les relations empiriques de Cambefort La figure 6 pour laquelle les déplacements de la tête du pieu sont égaux à ceux de tous les points du fât (pieu incompressible), nous permet de retrouver, comme l'a déjà fait Frank [13] dans le domaine "collé ", la règle de mobilisation du frottement latéral énoncée empiriquement par M. Cambefort :

$$
\begin{aligned}
& \tau=\alpha+\beta y \text { avant glissement } \\
& \tau=g+f z \text { après glissement }
\end{aligned}
$$

y étant le déplacement local du pieu au niveau $z ; \alpha, \beta, g, f$ des coefficients dépendant de la nature du sol et de la mise en place du pieu.

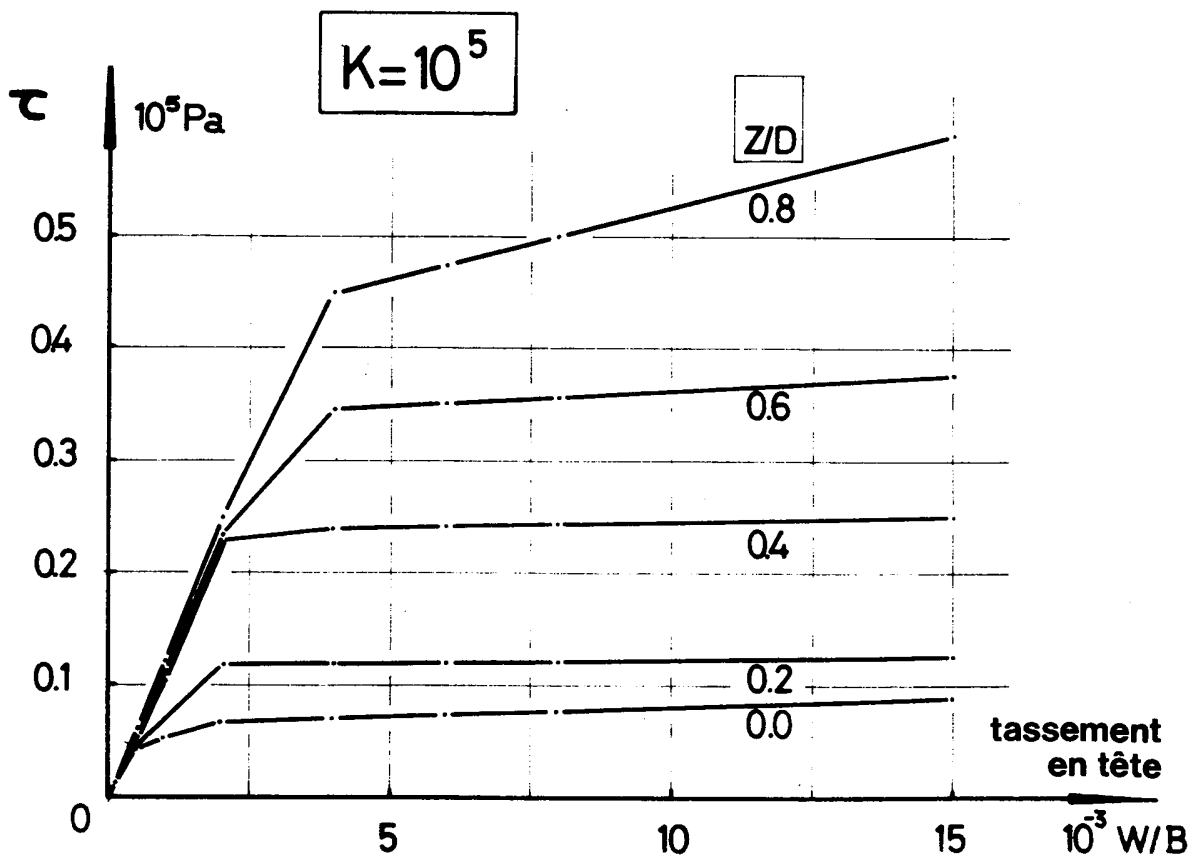

Fig. 6 Mobilisation calculée du frottement latéral local en fonction du tassement en tête, suivant la profondeur, $K=10^{5}$ $Z$ : profondeur du point considéré 


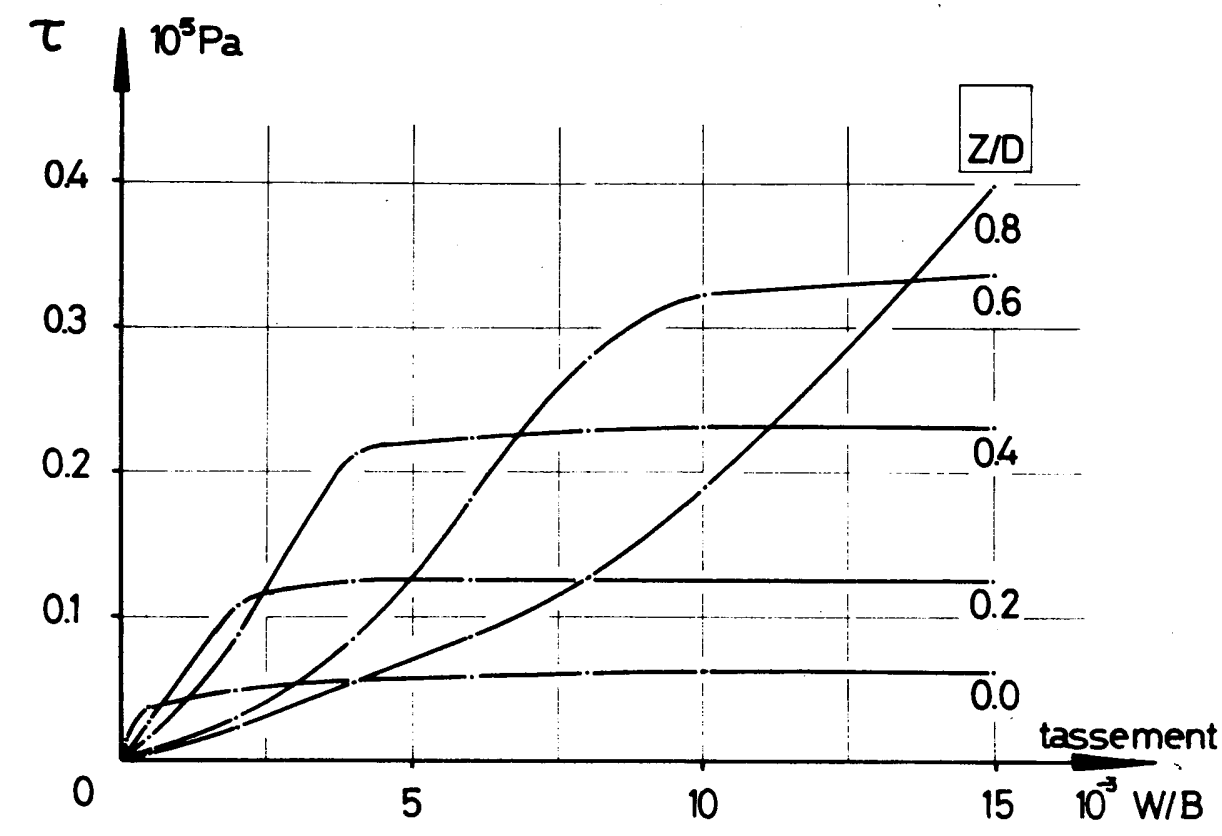

Fig. 7 Mobilisation calculée du frottement latéral local en fonction du tassement en tête, suivant la profondeur, $K=100$

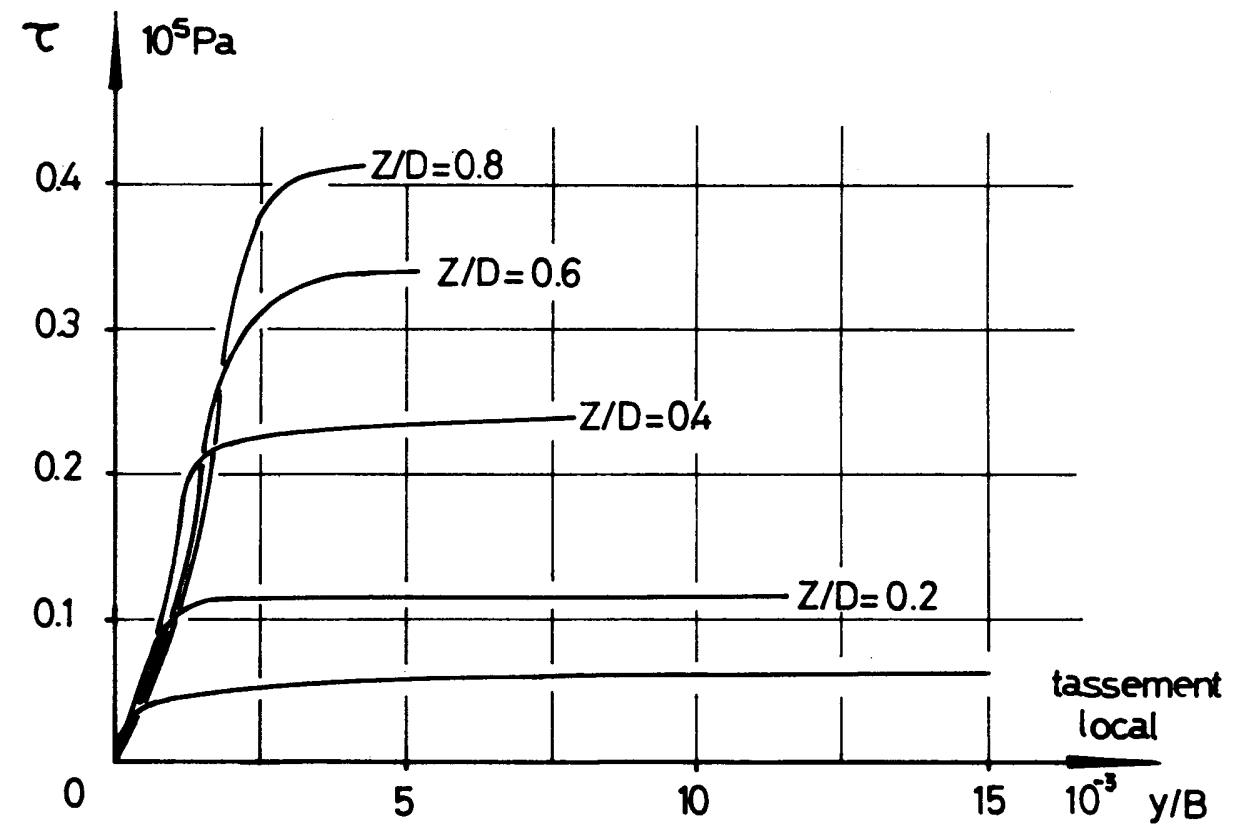

Fig. 8 Mobilisation calculée du frottement latéral local en fonction du tassement local, suivant la profondeur, $K=100$

Nous avons tracé figure 8 les résultats de la figure 7 en fonction des déplacement locaux du pieu (différents du déplacement de la tête dans le cas du pieu très compressible). On obtient alors une série de courbes proches de celles de la figure 6.

En comparant nos résultats avec les relations de Cambefort, on peut tirer les observations suivantes:

- Dans notre cas : $\alpha=0$ (pieu moulé, pas de contraintes résiduelles) $\mathrm{g}=0$ (milieu pulvérulent)

- Pour le pieu incompressible, $\beta$ est pratiquement constant.

- Pour le pieu compressible, $\beta$ n'est pas rigoureusement constant, ni en fonction de $y$, ni en fonction de $z / D$. On peut néanmoins déterminer une pente moyenne.

Avec les données de notre calcul $(D / B=30, B=50 \mathrm{~cm}$, $E=500.10^{5} \mathrm{~Pa}$ ) on trouve pour valeur moyenne de

$\beta=2,4.10^{5} \mathrm{~Pa} / \mathrm{cm}$ pieu incompressible

$\beta=2,8.10^{5} \mathrm{~Pa} / \mathrm{cm}$ pieu compressible

Exprimées en fonction de module, ces valeurs donnent :

$$
\beta=4,8.10^{2} \mathrm{E} \mathrm{Pa} / \mathrm{cm} \text { incompressible }
$$$$
\beta=5,6.10^{2} \mathrm{E} \mathrm{Pa} / \mathrm{cm} \text { compressible }
$$

On constate que ces relations sont très proches de celle donnée empiriquement par Cassan (9) pour les pieux forés :

$$
\beta=4,2 \cdot 10^{2} \mathrm{E} \mathrm{Pa} / \mathrm{cm}
$$

Elles sont également proches des valeurs mesurées par Baguelin et al [2].

- Dans la phase après glissement, on trouve bien une relation du type $\tau=f z$, avec fidentique dans les deux cas, égal à

$$
f=42 \mathrm{~Pa} / \mathrm{cm}
$$

Dans notre cas f est sensiblement égal à Ko $\gamma \operatorname{tg} \delta$ ( $\delta$ angle de frottement sol-pieu).

On peut donc affirmer que ces règles de mobilisation correspondent à l'introduction d'un frottement de type Coulomb au contact du pieu et d'un sol élastique. Par conséquent, les coefficients de Cambefort peuvent en première approximation être reliés aux paramètres élastiques du sol et au cœfficient de frottement sol-pieu. 


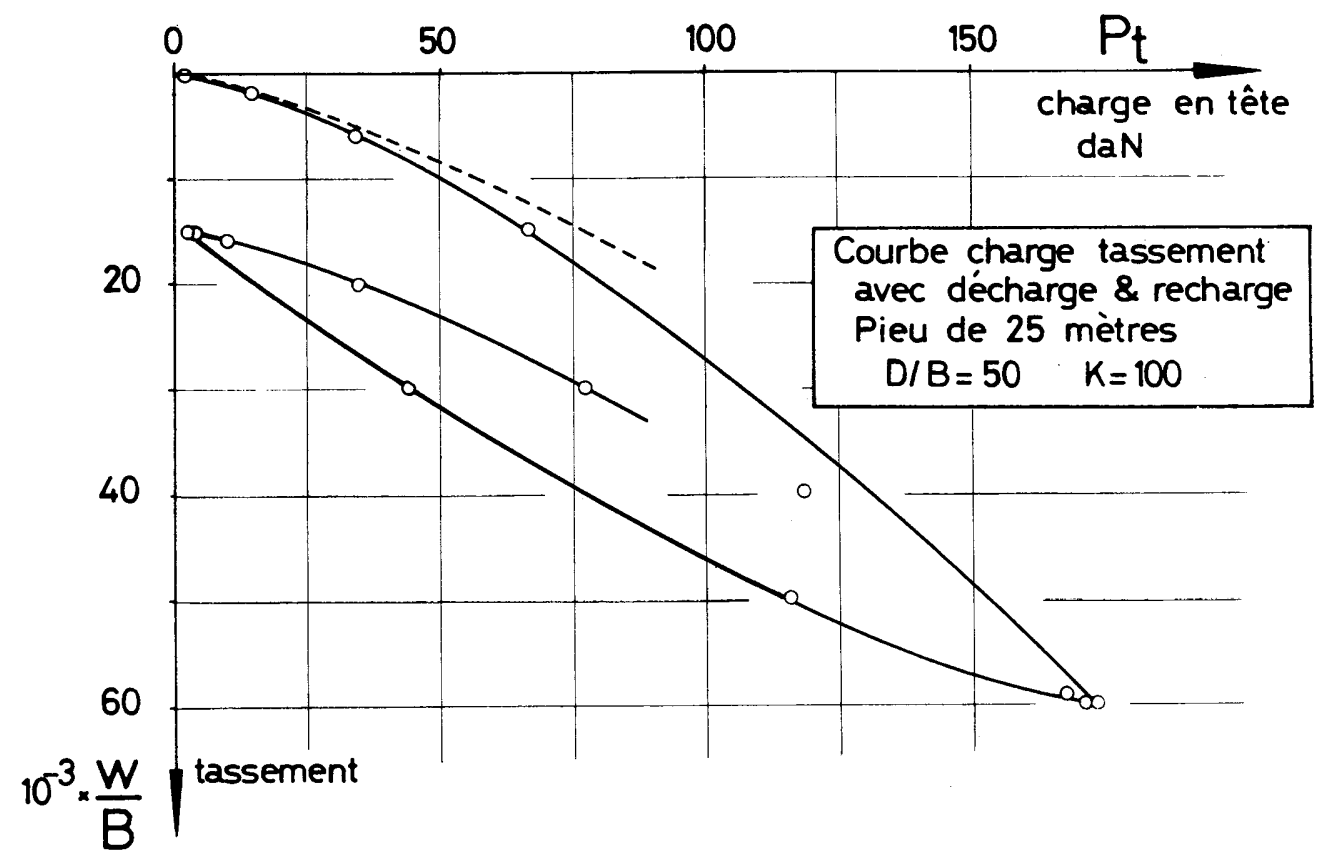

Fig. 9 Calcul d'un pieu soumis à une charge-décharge et recharge

Pieux subissant des sollicitations alternées

La figure 9 présente la courbe charge-tassement obtenue pour une sollicitation cyclique, composée d'une charge, d'une décharge et d'une recharge.

La courbe de recharge calculée présente une pente à l'origine tout à fait différente de la pente finale de la décharge; elle passe de façon très nette au-dessus de la courbe de décharge, ce qui est qualitativement en excellent accord avec les mesures expérimentales, et confirme nettement l'hypothèse suivant laquelle les contraintes résiduelles sont (pour partie) à l'origine de ces comportements constatés tant in situ qu'au laboratoire.

On remarque aussi que la courbe de tassement après décharge, reportée en pointillé sur la figure, a une pente plus faible que la courbe de tassement initiale. Ce résultat est remarquable car il montre que les contraintes résiduelles sont en partie responsables du raidissement de la courbe de tassement après décharge.

Nous avons porté sur la figure 10 les différentes répartitions de la contrainte de cisaillement le long du fât, au cours des trois phases du calcul. La compression des répartitions nécessite un examen détaillé; après la première phase de mobilisation progressive du frottement latéral le long du fût, avec glissement des points à l'interface (premier chargement, symboles blancs), on observe dans un deuxième temps l'inversion de la contrainte de cisaillement en haut du fât, et le développement en partie haute d'une zone de frottement négatif. Le maximum de la contrainte de cisaillement est atteint aux points les plus hauts, tandis qu'en partie basse le frottement latéral 'local reste positif et équilibre le frottement négatif. Le point neutre descend le long du fût.

Au cours de la troisième phase, de rechargement, la zone en frottement négatif est progressivement résorbée, et l'influence des contraintes résiduelles " s'efface " lorsque la répartition du frottement latéral atteint son profil avant déchargement.

Ces calculs montrent qu'il est possible, par une prise en compte correcte du glissement, de mettre en évidence l'apparition de contraintes résiduelles, et que celles-ci ont à elles seules une influence remarquable et conforme à l'expérience, sur les courbes charge-tassement. Ceci constitue un élément d'interprétation des différences constatées entre les essais de tassement sur pieu moulé, et les essais après fonçage. Bien entendu, la loi simple adoptée pour le sol ne permet néanmoins pas de tenir compte de l'écrouissage du sol.

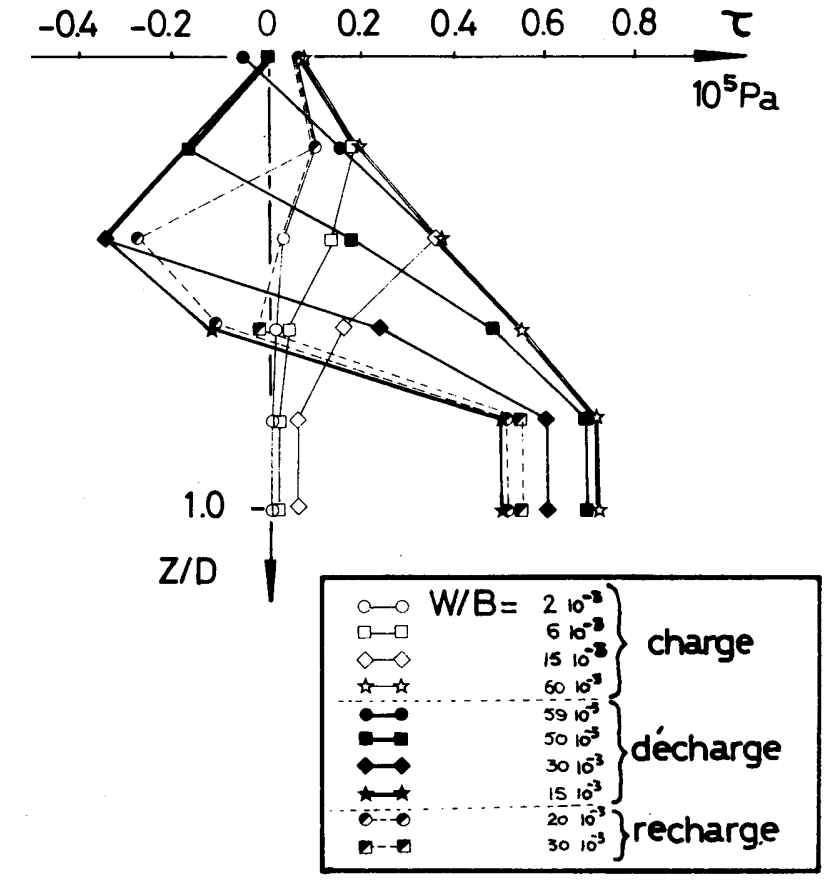

Fig. 10 Evolution de la répartition du frottement latéral lors d'une sollicitation alternée 


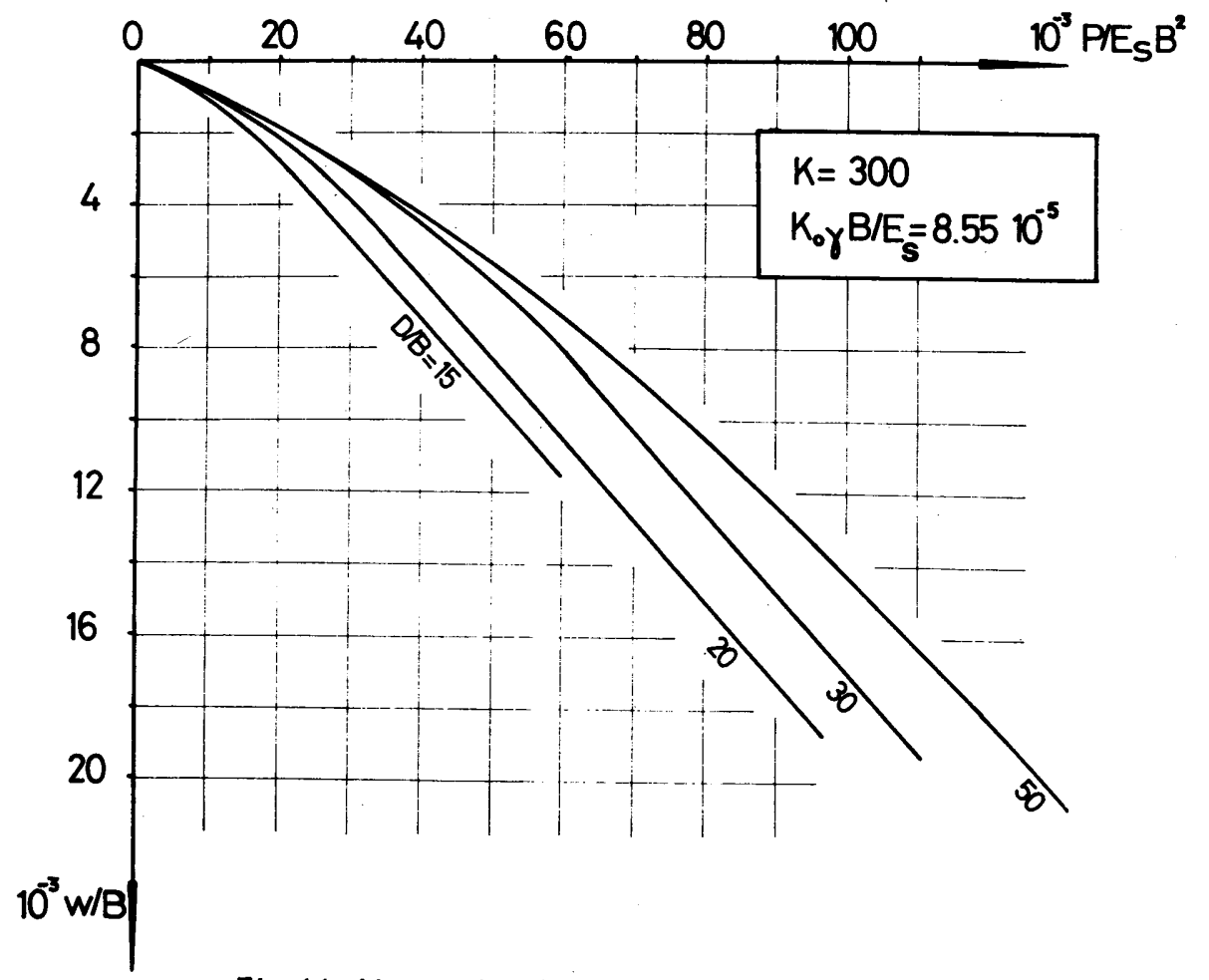

Fig. 11 Abaque de calcul du tassement $K=300$

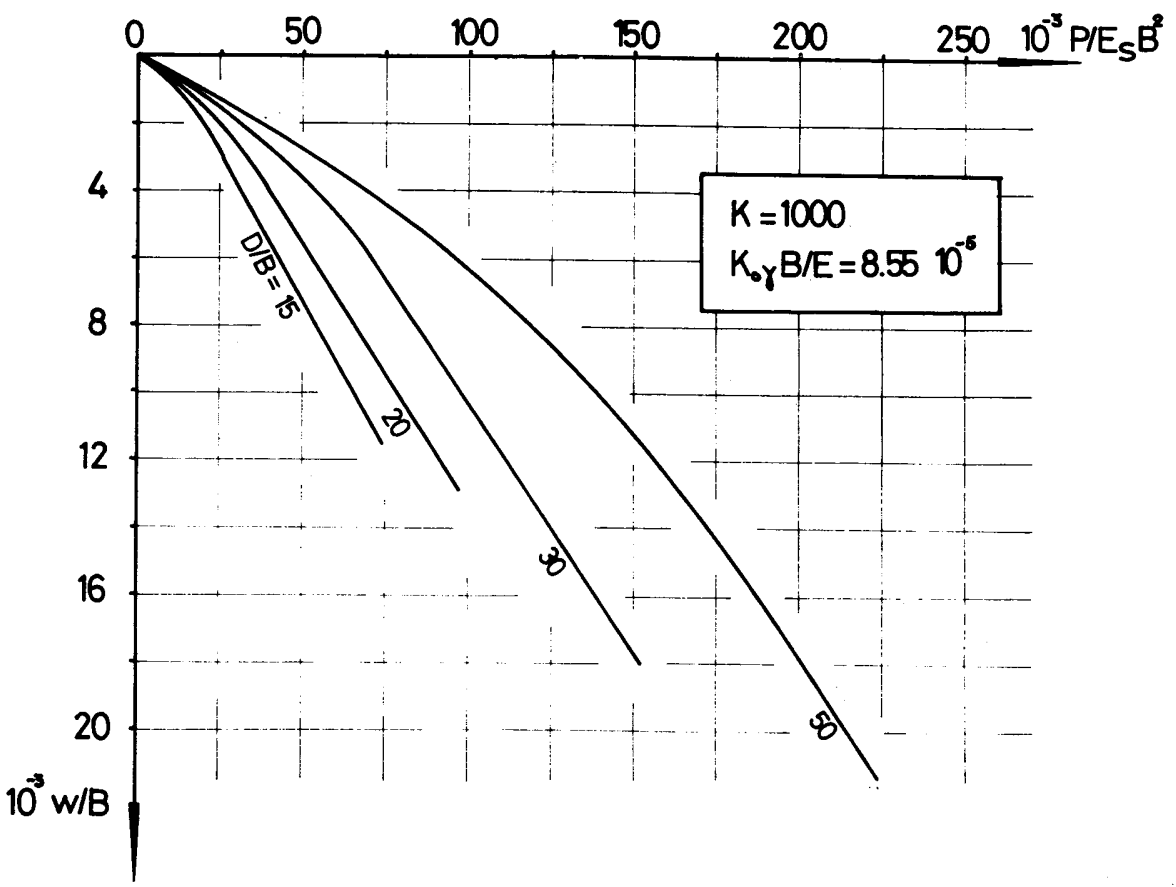

Fig. 12 Abaque de calcul du tassement $K=1000$

\subsection{Méthode pratique de calcul des tassements}

Nous présentons comme application de notre calcul quelques exemples d'abaques (fig. 11 et 12), permettant de calculer le tassement d'un pieu sous charge de service connaissant sa géométrie et les caractéristiques élastiques du sol. Bien entendu, ces calculs ne sont pas sans.limite, la modification des parametres du sol due au remaniement lors de la mise en place du pieu, étant mal connue, la simulation des contraintes résiduelles dues au battage ou au fonçage n'étant pas encore faite au niveau du calcul. Néanmoins, dans l'état actuel des connaissances et des moyens de calcul, ils constituent un progrès certain dans l'approche du tassement.
Les abaques donnent les courbes charge en tête-tassement d'un pieu, mises sous forme adimensionnelle, $P / E_{s} B^{2}=f(W / B)$, en introduisant :

- la compressibilité relative du pieu $K=E_{\text {pied }} E_{\text {sol }}$

$E_{\text {pieu }}$ : module d'Young du pieu

$E_{\text {sol }}$ : module d'Young du sol

- le paramètre « d'échelle » $K_{\circ} \gamma B E_{s}$

$\gamma$ densité du sol

$K_{0}$ coefficient de poussée des terres sur le pieu avant la mise en charge

B diamètre du pieu

Es module d'Young du sol 


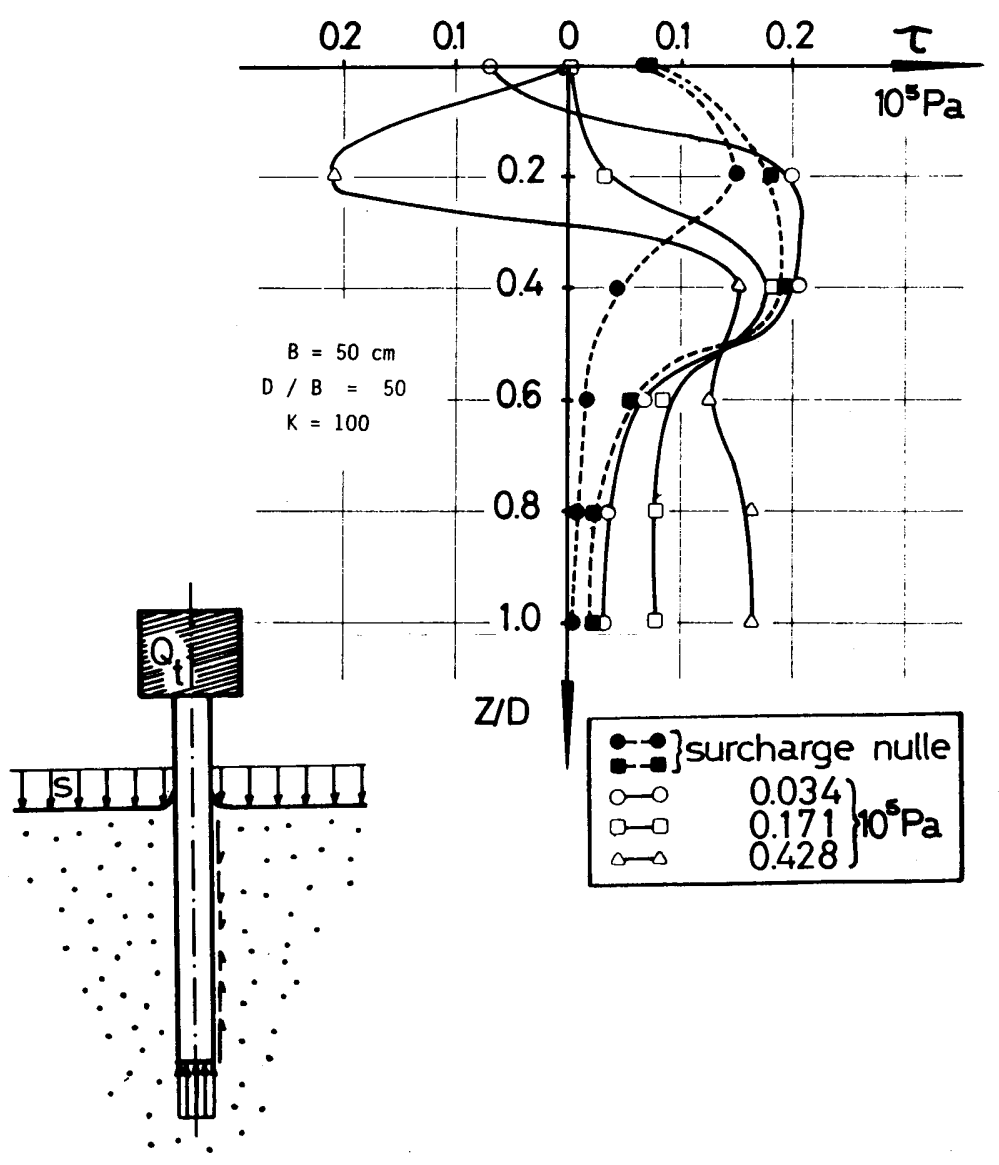

Fig. 13 Frottement négatif sur un pieu préalablement chargé à $40.10^{3}$ daN : calcul de l'effort dans le pieu en fonction de la surcharge

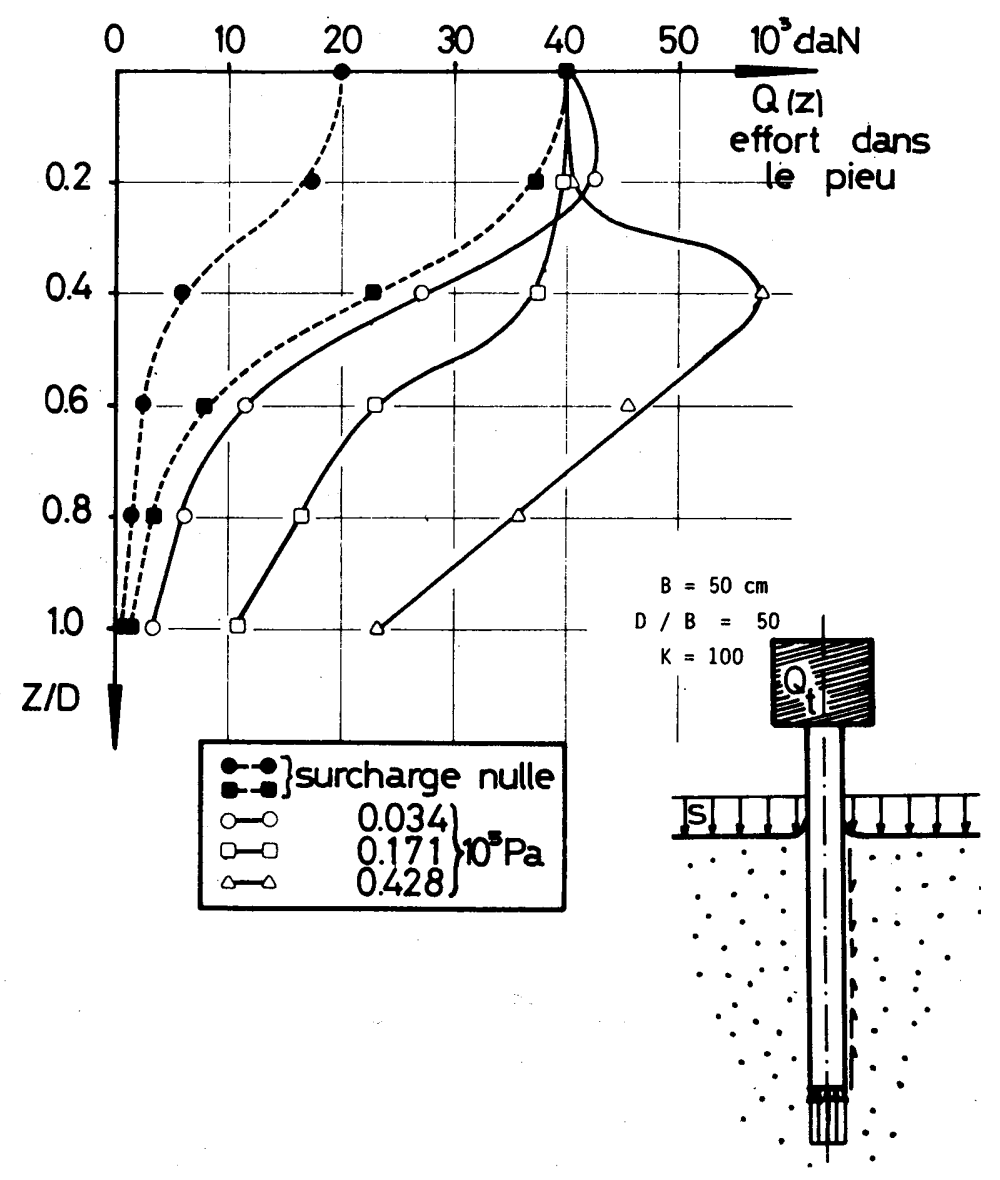

Fig. 14 Frottement négatif sur un pieu préalablement chargé à $40.10^{3}$ daN : calcul de la répartition des contraintes tangentielles en fonction de la surcharge 


\section{Frottement négatif}

\subsection{Généralités}

Dans le cas de frottement négatif, le calcul doit rendre compte de l'inversion des contraintes de cisaillement dans le haut du fût et déterminer l'accroissement correspondant de charge dans le pieu.

Les premiers calculs de frottement négatif ont été menés de façon plus ou moins empirique soit en cherchant la répartition des contraintes de cisaillement (Habib), soit en supposant que le cisaillement est maximum, égal à Ko $\gamma z \operatorname{tg} \varphi$ en un point courant du fût (Zeevaert).

L'utilisation de la méthode présentée ci-dessus pour le cas des pieux sous charge de service nous a permis d'étudier le problème du frottement négatif sans hypothèse particulière sur la valeur ou la répartition des contraintes de cisaillement ni sur la position du point neutre.

Nous présentons ici cette application au cas d'un pieu de diamètre $B=50 \mathrm{~cm}$, d'élancement $D / B=50$, de compressibilité $K=100$, installé sur un terrain pulvérulent de module $E_{S}=50 \mathrm{MPa}$, qu'on vient surcharger latéralement par un remblai qui exerce sur le sol une surcharge $s$ variable.

On considère le cas d'un pieu chargé en tête avec une charge de $20 \times 10^{3}$ et $40 \times 10^{3}$ daN.

\subsection{Résultats}

La figure 13 indique l'évolution de la charge dans le pieu. Les courbes en pointillé indiquent la répartition sous les charges de service de $20 \times 10^{3}$ et $40 \times 10^{3}$ daN. On voit que dans ces conditions, seule la moitié supérieure du pieu est mobilisée et que la pointe ne travaille pas.

Lorsqu'on applique des surcharges latérales faibles, on observe peu de modification sur la forme générale de la courbe, mais une mobilisation progressive de la pointe. Pour une surcharge forte par contre, on a apparition d'un pic où l'effort dans le pieu est nettement supérieur à l'effort appliqué en tête.

Sur la figure 14 qui montre l'évolution des contraintes de cisaillement le long du pieu, on observe une répartition de contraintes partagée : contrainte négative dans le haut du pieu, et positive dans le bas. Le "point neutre ", où $\tau(z)$ change de signe correspondant à celui où l'effort $Q(z)$ est maximum. La position du point neutre dépend de la valeur de la surcharge appliquée.

\section{Conclusion}

Les calculs présentés ici montrent l'importance de la loi de contact entre le sol et le pieu, ainsi que la souplesse de l'application de la méthode à des cas de sollicitations variées pour les pieux : tassement sous charge verticale, monotone ou alternée, frottement négatif avec pieu fixé en tête ou libre en tête. D'autres applications ont été effectuées, en particulier le cas du pieu sollicité à l'arrachement.

L'introduction du glissement dans le calcul permet, tout en supposant le reste du massif élastique linéaire, de rendre compte des nombreux mécanismes de mobilisation du frottement latéral qu'un simple calcul "collé»" ne permet pas de décrire: non linéarité des courbes chargetassement, non réversibilité, transfert de charge du fât vers la pointe du pieu, apparition de contraintes résiduelles lors d'une décharge.

Certes, le fait de se limiter à l'élasticité linéaire pour le sol ne permet pas de décrire tout le problème du tassement. comme l'écrouissage du sol lors d'une recharge ou l'évolution du module avec la contrainte moyenne appliquée. De plus, la simulation numérique des conditions aux limites initiales reste délicate (prise en compte du remaniement et des contraintes résiduelles dues à la mise en place). Nous avons effectué une approche dans ce sens au cours d'autres travaux [5], en utilisant à la fois une loi incrémentale pour le sol et le frottement-glissement au contact du pieu. Si elle est plus séduisante, la mise en œuvre de cette seconde étape est plus lourde et le calcul doit être envisagé cas par cas.

La méthode présentée dans cet article constitue un progrès très net dans la mesure où elle nous a permis d'étudier un grand nombre de cas et d'aboutir à l'élaboration d'abaques de calcul facilement utilisables par le praticien.

\section{Références Bibliographiques}

[1] BUISSON, AHU, HABIB. - Le frottement négatif. Supplément aux Annales de I'ITBTP, janvier 1960.

[2] BAGUELIN, BUSTAMANTE, FRANK, JEZEQUEL. - La capacité portante des pieux. Annales de l'ITBTP $n^{\circ} 330$, juillet-aoat 1975.

[3] BOOKER, POULOS. - Analysis of Creep Settlement of Pile Foundations. Journal of Geotechnical Engineering Division, ASCE, January 76.

[4] BOULON, CHAMBON, DARVE, FORAY, MORELOT, PUECH, ZINEBI. - Tassement et Force portante limite des pieux en milieu pulvérulent. $6^{\circ}$ Congrès Européen de Mécanique des Sols et des Travaux de Fondations, Vienne, Vol. 1.2, pp. 337, 1976.

[5] BOULON, CHAMBON, DARVE, DESRUES, FLAVIGNY, FORAY. - Comportement d'un écran et d'un pieu : expérience et calcul. 9th ICSMFE, Tokyo, 1977.

[6] BOULON, DARVE, DESRUES, FORAY. - Traitement numérique des problèmes a loi rhéologique et conditions aux limites non linéaires en mécanique des sols. International Symposium of Innovative Numerical Analysis in Applied Engineering Science. Versailles, mai 1977, à publier dans la revue «Computers and Structures 》.

[7] BUTTERFIELD, BANERJEE. - The Elastic Analysis of Compressible Piles and Pile Groups. Géotechnique $21, n^{\circ} 1$, 1971.

[8] CAMBEFORT. - Essai sur le comportement en terrain homogène des pieux isolés et des groupes de pieux. Annales de I'ITBTP, N²04, décembre 1964.

[9] CASSAN. - Les essais in-situ en Mécanique des Sols. Tome 2 : Application et Méthodes de Calcul. Eyrolles édit.

[10] DESAl. - Numerical design-analysis for piles in sand. Journal of the Geotechnical Engineering Division, ASCE, June 1974.

[11] DESRUES. - Contribution l'etude du tassement des fondations profondes. Thèse de Docteur-Ingénieur, Université I de Grenoble, mars 1977.

[12] ELLISON, D'APPOLONIA, THIERS. - Load-deformation Mechanism for bored Piles. Journal of the Soil Mechanics and Foundations Division, ASCE 97, $n^{\circ}$ SM4, April 71.

[13] FRANK. - Etude théorique du comportement des pieux sous charge verticale. Introduction de la dilatance. Rapport de recherche $n^{\circ} 46$, Laboratoire des Ponts et Chaussées, Ministère de l'Équipement 1975.

[14] POULOS. - Load-sett/ement Prediction for Piles and Piers. Journal of the Soil Mechanics and Foundation Division ASCE, Sept. 72.

[15] REESE. - Load versus settlement for an axially loaded Pile. Symposium on Bearing Capacity of Piles, Central Building Research Institue, Roorkee (India), February 1974.

[16] TERZAGHI, PECK. - Mécanique des Sols Appliquée. Dunod, Paris 1970.

[17] THURMAN, D'APPOLONIA. - Computed Movement of Friction and End-Bearing Piles embedded in Uniform and Stratified Soils. 6th ICSMFE Montreal, p. 323, 1965.

[18] VESIC, HOLLOWAY, WAYNE, CLOUGH. - The Mechanics of Pile-Soil Interaction in Cohesionlesssoil. Soil Mechanics, Series $n^{\circ} 39$, School of Engineering Duke University 1975.

[19] ZEEVAERT. - Reduction of Point Bearing Capacity of Piles because of Negative Friction. $1 \mathrm{e}$ Congreso Panamericano de Mecanica de Suelos y Cimentaciones, Mexico 1959.

[20] ZINEBI. - Contribution à l'étude des problèmes à symétrie de révolution par la méthode des eléments finis. Thèse de Docteur-Ingénieur, Université I de Grenoble, juin 1975. 